\title{
Knee arthroscopy: the science of art
}

\author{
John Nyland ${ }^{1}$ David N. M. Caborn ${ }^{2} \cdot$ Roland Jakob $^{3}$
}

Received: 26 June 2015 / Accepted: 8 July 2015 / Published online: 22 July 2015

(C) European Society of Sports Traumatology, Knee Surgery, Arthroscopy (ESSKA) 2015

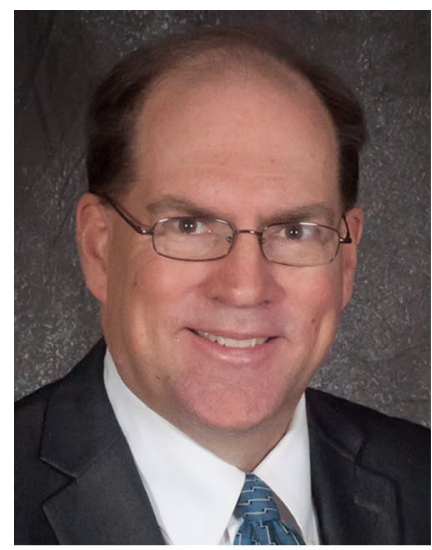

John Nyland

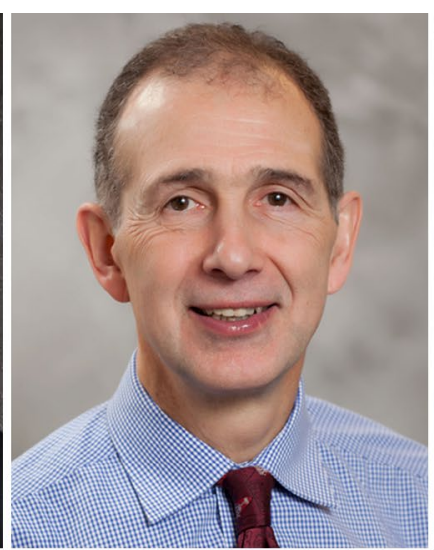

David N. M. Caborn

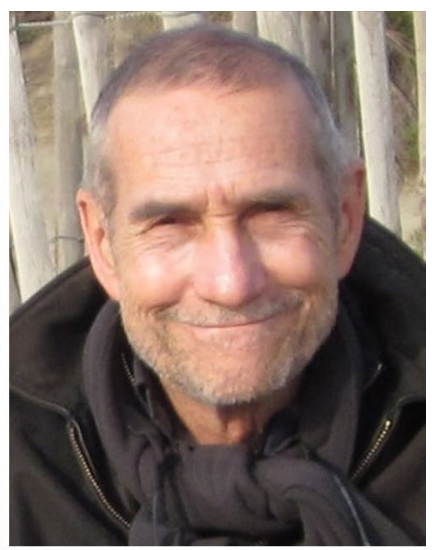

Roland Jakob
At a recent knee meeting, many interesting topics were discussed including preservation of the ACL remnant during reconstruction, graft selection, use of innovative synthetic or culture-grown grafts, and articular cartilage preservation or repair procedures [1-3]. The research methodologies used were as diverse as the countries of origin of the participating surgeons and rehabilitation professionals. Despite obvious efforts to maintain scientific rigour, the impression instilled in us was that knee surgery will always be an art first and a science second.

In touring an art gallery, the viewer may have the opportunity to observe the work of talented artists with diverse

John Nyland

jnyland@spalding.edu

Spalding University, Louisville, KY, USA

2 Shea Orthopedic Group, KentuckyOne Health, Louisville, KY, USA

3 Orthopadie FMH, 1787 Môtier (Vully), Switzerland styles such as Rembrandt, Matisse, Renoir, Van Gogh, Monet, Da Vinci, Michelangelo, or Picasso. Although the paint and canvas used by each artist as well as the perceived quality of the end work may be similar, the technique employed and its appearance differ considerably in style and artistic approach. To the knee surgeon, each patient may be a new living canvas and the paint and brushes may be the tools used to create the reparative or reconstructive art given a specific set of indications. Patients differ considerably with regard to previous medical and surgical experiences, age, expectations of surgical or rehabilitation outcomes, psycho-behaviours, sporting interests, and many other variables. Using this simple analogy, no two living canvases are alike! What can and should the surgical artist do to optimize how they interact with each patient? This may represent the natural and regular dilemma that exists when we consider how best to appraise or measure the value of this "surgical" art [4]. How effectively can a measurable context be applied that truly evaluates the ability of both the surgeon to create, and the patient to adhere to 
recommendations in an effort to achieve high value in the form of a successful outcome? How comprehensively do we realize or measure the subtle tones, textures, and stroke patterns which may reflect the perceived abilities, objective qualitative and quantitative appraisal of function, reduced pain, psycho-behavioural characteristics, educational goal achievements, and quality-of-life considerations for each patient.

While the surgeon tends to focus on the tools and their proper use [5], they should be similarly motivated to accurately appraise the true value of the art that is created. Ultimately, it is the second set of tools that necessitate greater attention regarding the need for scientific innovation and greater validity. But can the surgeon, or for that matter the patient accurately judge the end result in a highly objective manner? For this reason, it is essential that they cultivate a team of associates or consultants who understand both the conceptual basis and indication of the intervention. This team must also maintain the objectivity needed to accurately appraise the finished work and perhaps the influence of the patient's actions or lack thereof. Can the measurement tools be refined in terms of accuracy, the precise construct being measured, and how well they discriminate the "beauty" or value of the art? How effectively did the surgeon meet patient expectations and identify indications regarding the need to achieve certain goals or desires that were communicated at the onset of the work? How compliant was the patient with the surgeon's recommendations, adhering to the agreed upon psycho-behavioural change requests necessary for the surgery and rehabilitation to be effective? Only with this information will the surgical artist truly know the appraised value of their work for each living canvas. An associated question is how soon after the creation of the art should it be appraised? Should it be 2, 5, 10, or 20 years afterwards? Does the value of the art continue to appreciate? What is the true end goal that best reflects the restoration of patient function within the influence of ageing and associated activity modifications? There must be a balance between early and delayed gratification for both the surgeon and the patient. Both the surgeon and the patient likely need to change how they appraise the value of the art that has been created. Perhaps greater attention should be placed on the indications that drive the surgeon's decisions in addition to greater accuracy and relevance in the methods used to measure treatment efficacy both in surgery and rehabilitation. The surgeon must compile, screen, and interpret data from clinical examination, imaging, medical and surgical history, genetic disposition, patient expectations, lifestyle, and quality-of-life values to propose a plan of care. From this confluence of information, the surgeon compares and contrasts perceptibly strong indications, strong contraindications, and somewhat equivocal findings. The process by which this information is carefully gathered and interpreted is very much an art. In the end, it is impossible to separate the artist from the art, or the art from the "living canvas".

\section{References}

1. International Cartilage Repair Society 2015 Meeting. May 8-11, 2015, Chicago, Illinois, USA

2. Summit of Asia-Pacific Knee, Arthroscopy and Sports Medicine Society Meeting. May 8-9, 2015, Taipei, Taiwan

3. The Art and Science of Sports Medicine Conference. June 1-5, 2015, Curry School of Education, University of Virginia, Charlottesville, Virginia, USA

4. Russo R (2015) Art and science a unique world. Knee Surg Sports Traumatol Arthrosc 23:629-635

5. Espregueira-Mendes J, Karahan M (2014) Orthopaedic sports surgery: art or science? Knee Surg Sports Traumatol Arthrosc 22:959-960 